\title{
RNA-Seq analysis of blood meal induced gene-expression changes in Aedes aegypti ovaries
}

Dilip K. Nag ${ }^{1 *}$ D, Constentin Dieme ${ }^{1}$, Pascal Lapierre ${ }^{2}$, Erica Lasek-Nesselquist ${ }^{2,3}$ and Laura D. Kramer ${ }^{1,3}$

\begin{abstract}
Background: Transmission of pathogens by vector mosquitoes is intrinsically linked with mosquito's reproductive strategy because anautogenous mosquitoes require vertebrate blood to develop a batch of eggs. Each cycle of egg maturation is tightly linked with the intake of a fresh blood meal for most species. Mosquitoes that acquire pathogens during the first blood feeding can transmit the pathogens to susceptible hosts during subsequent blood feeding and also vertically to the next generation via infected eggs. Large-scale gene-expression changes occur following each blood meal in various tissues, including ovaries. Here we analyzed mosquito ovary transcriptome following a blood meal at three different time points to investigate blood-meal induced changes in gene expression in mosquito ovaries.
\end{abstract}

Results: We collected ovaries from Aedes aegypti that received a sugar meal or a blood meal on days 3, 10 and 20 post blood meal for transcriptome analysis. Over 4000 genes responded differentially following ingestion of a blood meal on day 3, and 660 and 780 genes on days 10 and 20, respectively. Proteins encoded by differentially expressed genes (DEGs) on day 3 include odorant binding proteins (OBPs), defense-specific proteins, and cytochrome P450 detoxification enzymes. In addition, we identified 580 long non-coding RNAs that are differentially expressed at three time points. Gene ontology analysis indicated that genes involved in peptidase activity, oxidoreductase activity, extracellular space, and hydrolase activity, among others were enriched on day 3. Although most of the DEGs returned to the nonsignificant level compared to the sugar-fed mosquito ovaries following oviposition on days 10 and 20, there remained differences in the gene expression pattern in sugar-fed and blood-fed mosquitoes.

Conclusions: Enrichment of OBPs following blood meal ingestion suggests that these genes may have other functions besides being part of the olfactory system. The enrichment of immune-specific genes and cytochrome P450 genes indicates that ovaries become well prepared to protect their germ line from any pathogens that may accompany the blood meal or from environmental contamination during oviposition, and to deal with the detrimental effects of toxic metabolites.

Keywords: Aedes aegypti, RNA-Seq, Differential gene expression, Blood meal, Egg development

* Correspondence: dilip.nag@health.ny.gov

${ }^{1}$ Arbovirus Laboratory, Wadsworth Center, New York State Department of Health, Slingerlands, NY 12159, USA

Full list of author information is available at the end of the article

(c) The Author(s). 2021 Open Access This article is licensed under a Creative Commons Attribution 4.0 International License, which permits use, sharing, adaptation, distribution and reproduction in any medium or format, as long as you give appropriate credit to the original author(s) and the source, provide a link to the Creative Commons licence, and indicate if changes were made. The images or other third party material in this article are included in the article's Creative Commons licence, unless indicated otherwise in a credit line to the material. If material is not included in the article's Creative Commons licence and your intended use is not permitted by statutory regulation or exceeds the permitted use, you will need to obtain permission directly from the copyright holder. To view a copy of this licence, visit http://creativecommons.org/licenses/by/4.0/ The Creative Commons Public Domain Dedication waiver (http://creativecommons.org/publicdomain/zero/1.0/) applies to the data made available in this article, unless otherwise stated in a credit line to the data. 


\section{Background}

Mosquito-borne pathogens are responsible for some of the widespread infectious diseases worldwide, such as malaria, encephalitis, filariasis, dengue fever, and yellow fever $[1,2]$. Since there are no antiviral drugs or safe and effective FDA-approved vaccines against several medically important pathogen-associated ailments, vectorcontrol strategies remain the only effective route to prevent a disease outbreak. Consequently, mosquitoes became the object of intensive investigations in order to develop novel vector-control strategies. The availability of the mosquito genome sequence provides an excellent opportunity to identify host gene targets to control pathogen transmission.

For anautogenous mosquitoes, the vector competence for transmitting a pathogen is essentially linked with their reproductive strategy, as the female normally depends on a vertebrate blood meal as a source of nutrition to produce a batch of eggs [3]. The cycle of blood feeding, egg development, and egg laying is collectively known as the gonotrophic cycle. After each gonotrophic cycle, mosquitoes return to their host-seeking stage for another blood meal. Mosquitoes that acquire pathogens during the first blood meal may transmit the pathogen to an uninfected host during these subsequent blood meals. In addition to this horizontal mode of transmission, with some viruses, the pathogens can be transmitted vertically to progeny via infected eggs [4-12]. Vertical transmission becomes important for pathogen maintenance during adverse environmental conditions, or when the number of susceptible vertebrate hosts is rare due to herd immunity or vaccination.

In Aedes aegypti, an anautogenous mosquito, the preblood meal period in the first gonotrophic cycle also includes the post-eclosion development period, which persists from $72 \mathrm{~h}$ to until the uptake of the first blood meal. Oogenesis in the mosquito ovary begins posteclosion, but the oocyte growth is attenuated at a resting stage until the ingestion of a blood meal after which egg development continues until oviposition (i.e., egglaying). In the post blood-meal (PBM) period, mosquitoes use about $20 \%$ of the blood nutrients to produce egg components within $48 \mathrm{~h}$ and another fraction to carry out intense biosynthetic activities, then excrete the rest $[13,14]$. It takes about $72 \mathrm{~h}$ to complete the egg development during the PBM period. Protein-rich blood meal is required for oocyte development and vitellogenesis, during which yolk constituents (both protein and lipid) generated in the fat body are taken up by oocytes for storage and later use during embryogenesis. Vitellogenesis and oogenesis require a high level of coordination of molecular events in the fat body and ovary [3]. Multiple hormones are involved in this coordination process. Newly emerged females produce a large amount of juvenile hormone, which prime the fat body for the synthesis of vitellogenin, the precursor to the major yolk protein vitellin, and initiate limited ovarian follicle growth to its pre-vitellogenic resting stage [15]. A blood meal triggers the release of ecdysone by the ovaries; fat body cells take up ecdysone and convert it to 20-E, which triggers the activation of transcription of vitellogenin genes, coding for egg-yolk proteins, and other genes, the products of many of them are incorporated into eggs [16-18].

Clearly, a complex series of physiological events occurs in multiple tissues (e.g., midgut, fat body, and ovary) following blood meal ingestion. RNA-Seq analysis provides a useful tool to analyze changes in gene expression in the whole organism as well as in pertinent tissues [19, 20]. Comparing gene expression patterns at various time points between sugar-fed and blood fed mosquitoes and tissues, one can identify the organism's or tissue-specific responses to the blood meal. Previous studies used RNA-Seq, microarray, and EST analyses to identify differentially expressed genes in response to blood feeding in Anopheles gambiae, A. aegypti, and Aedes albopictus mosquitoes and in tissues, such as midgut and reproductive tissues [14, 21-29]. Similar approaches have also been used to investigate the mosquito's response to pathogen infection by several investigators [30-41]. Here, we used RNA-Seq to analyze differential gene expression following a blood meal at three time points (Days 3, 10, and 20) in A. aegypti ovaries without eggs. Previous transcriptome analyses in $A$. aegypti ovaries were carried out at various time points PBM until 72$96 \mathrm{~h}$ (i.e., the duration of the gonotrophic cycle) and also during embryonic development. In these studies, gene expression at late time points in the gonotrophic cycle was monitored in gravid ovaries. Here, we analyzed gene expression in ovaries without the eggs. Mosquitoes are expected to return to the pre-blood meal stage following each gonotrophic cycle. Our results indicated that although gene expression patterns following the gonotrophic cycle at late time points do not completely match with that of the non-blood fed (i.e., sugar fed) control mosquito ovaries, most differentially expressed genes (DEGs), however, return to the sugar-fed control level. In addition, several detoxification and defensespecific genes are also expressed at the early time point, suggesting that ovaries become prepared to avoid the ill effects of the blood meal derived toxic metabolites or to effectively deal with the pathogens that may accompany the blood meal.

\section{Results and discussion}

RNA-Seq analysis of $A$. aegypti ovary transcriptome

We carried out experiments to determine the ovaries' response to blood meal ingestion by RNA-Seq analysis. 
Mosquitoes were fed with sheep blood and engorged mosquitoes were separated in cardboard containers. Sugar-fed mosquitoes were used as controls. Ovaries were collected from sugar-fed (SF) control and bloodfed (BF) mosquitoes on days 3, 10, and 20 PBM. In our experiments, mosquitoes were allowed to lay their eggs by providing ovitraps following blood feeding. Consequently, by day 10 , most mosquitoes in the blood-fed group had laid their eggs and returned to the nongonotrophic stage, similar to SF females. However, in several mosquitoes there were one or few unlaid eggs in the ovaries. They were manually removed before ovary collection. RNA sequencing was carried out with total RNA extracted from pooled ovaries from SF and BF mosquitoes using Illumina sequencing technology. The above three time points were selected to determine changes in gene expression patterns during and after the gonotrophic cycle. A total of 19 samples (18 samples from three biological replicates and one additional sample (day 3 BF sample) from another independent replicate, see Methods) were sequenced. Bioinformatics analysis was carried out using the CLC genomics workbench. The total number of reads per sample varied between 48,669,332 and 72,981,770 among the 19 sequenced RNA samples (Suppl. Table 1). More than $77 \%$ of the reads mapped to the host genome, with about $94 \%$ mapping to the gene regions and $6 \%$ to the intergenic regions (Suppl. Table 1).

We carried out a principal component analysis (PCA) of SF and BF libraries to examine the clustering of data based on ingestion of a sugar meal or a blood meal. All biological replicates of SF and BF samples were distributed in two distinct groups (Fig. 1). Differential gene expression analysis indicated that in all time points, there were 5729 DEGs, with day 3 samples having the maximum number of DEGs (4289), and 249 DEGs were common to all three time points (Figs. 2 and 3). The numbers of DEGs on days 10 and 20 were similar (660 and 780 , respectively) (Fig. 3). On day 3 , there were 2743 DEGs with FDR $p$-value of $<0.05$ and $\log _{2}$ fold changes $>1$ (Suppl. Table 2). Under similar criteria, the number of DEGs on day 10 and 20 were 363 and 436, respectively. We have compared our RNA-Seq results with those of the previously reported transcriptome analyses of $A$. aegypti ovaries [25, 27]; the results are shown in Supplementary Table 2 and discussed below.

\section{Nature of DEGs in mosquito ovaries at different time points following blood meal ingestion}

Since day 3 PBM had the most DEGs, we, first, focused on the nature of genes that showed differential expression patterns at this time point. Most of the DEGs are not characterized. However, we observed that several groups of genes showed altered expression patterns. One interesting group consists of odorant-binding proteins (OBPs). The term 'odorant-binding proteins' is used to refer to a large family of insect proteins that are exceptional in their number, abundance and diversity. The name derives from the expression of many family members in the olfactory system of insects; OBPs are involved in detection of odors and translocation of volatile chemicals to the molecular components of the olfactory receptor neuron dendritic membrane, such as odorant receptors, gustatory receptors and ionotropic receptors, which are involved in odorant recognition and transduction of volatiles into electric signals [42, 43]. Among the 13 differentially expressed OBPs, only one had a 13-fold reduction in expression over the SF control, and the rest showed overexpression ranging from 2 to 244-fold (Table 1). Many odorant receptors also had differential expression patterns (Suppl. Table 2).

Previously, Akbari et al. [25] and Matthews et al. [27] studied gene-expression patterns in $A$. aegypti ovaries at various time points until $96 \mathrm{~h}$ PBM. Several genes that exhibited differential expression patterns in gravid ovaries were also differentially expressed in our system (Suppl. Table 2). Akbari et al. [25] also noted highly enriched OBPs PBM (Suppl. Table 2). However, highly overexpressed OBPs were not observed by Matthews et al. [27]. We expected some differences between the two studies, as mosquitoes in their system had no access to water to oviposit [27], whereas in our case a significant number of mosquitoes had laid their eggs at the time of sample collection, and eggs, if present, were removed from the ovaries before collection. Additionally, there were differences in the time (72 vs $96 \mathrm{~h}$ ) of sample collection. It is also possible that some differences in expression patterns between the current study and previous studies are be due to geographic origin of mosquito strains [Mexico vs Africa (Liverpool strain)] used in these two studies. It has been shown that significant changes in gene expression patterns occur in Aedes strains depending on the place of origin, number of generations in the laboratory, and susceptibility to dengue infection [44].

During a gonotrophic cycle, after a blood meal, the host-seeking behavior is decreased and at the same time mosquitoes' ability to find a suitable oviposition site is increased. This is when the females are behaviorally attracted to potential oviposition sites and the associated olfactory cues. Therefore, upregulation in the expression of olfactory receptors that are more attuned to oviposition attractant compounds and downregulation of receptors that are involved in recognition of compounds for host-seeking behavior in the antenna of $A$. aegypti PBM $[24,27]$ is not surprising. Our results showed that several odorant receptors (Or121, Or122, Or117, Or113, and Or6) were upregulated and Or30 was downregulated 


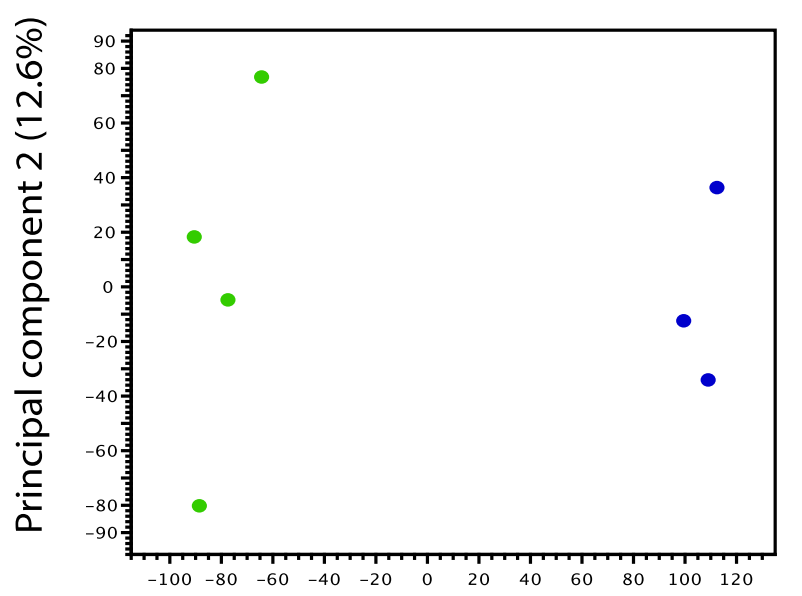

Day 3

Principal component 1 (49.6\%)

- BF

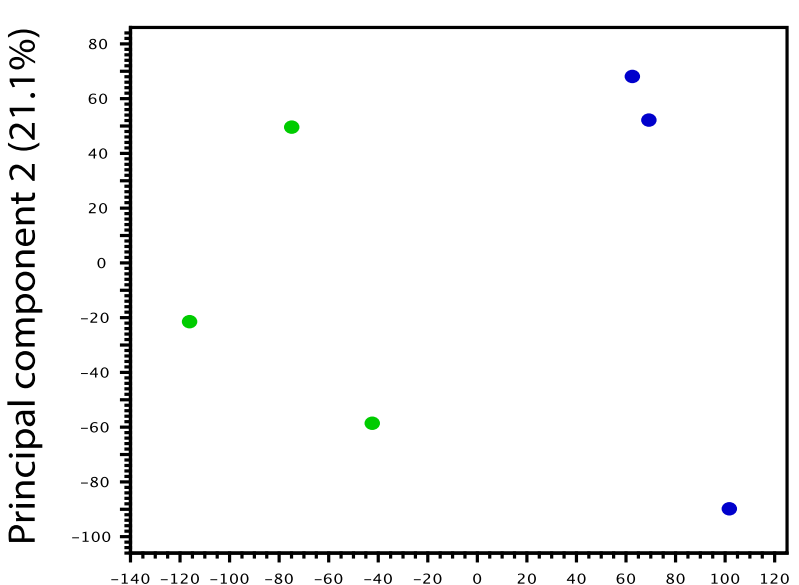

- SF

Day 10

Principal component 1 (38.7\%)

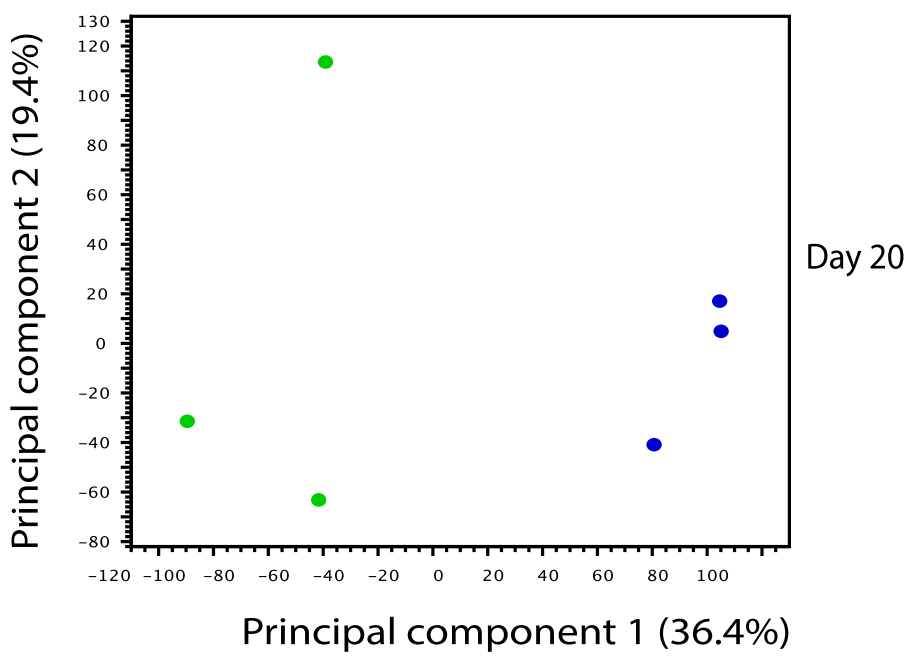

Fig. 1 Principal component analysis of the ovary RNA-Seq data. The samples were collected at three different time points from sugar fed (SF) and blood fed (BF) mosquitoes. A total of 19 samples were analyzed by RNA-Seq (see Methods) 


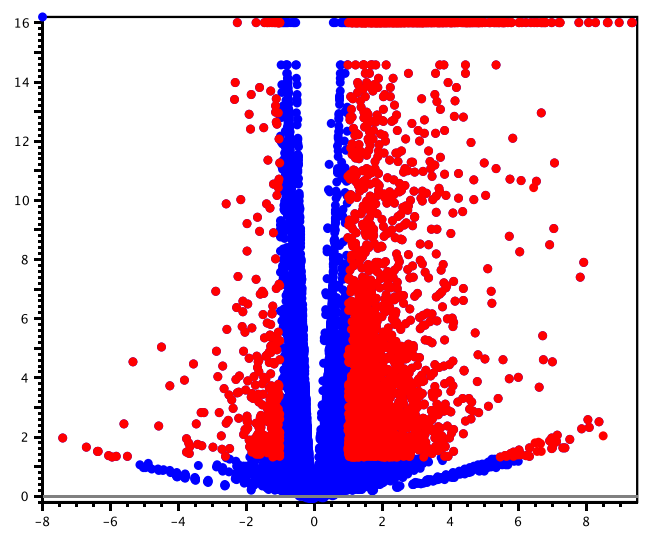

Day 3

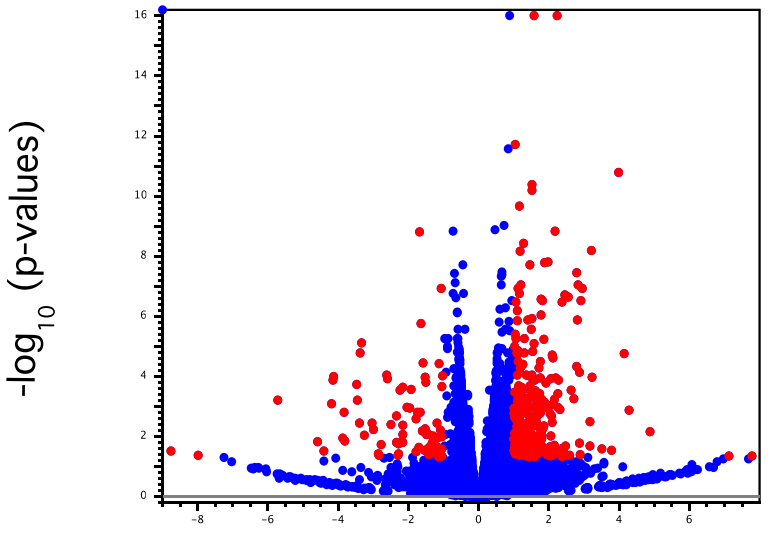

Day 10

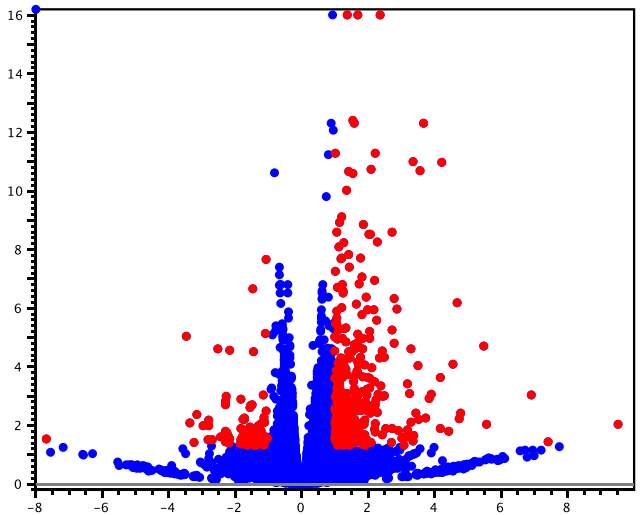

Day 20

\section{$\log _{2}$ fold change}

Fig. 2 Volcano plot analysis of differentially expressed genes (DEGs) between blood fed (BF) and sugar fed (SF) ovary tissues. Red circles indicate DEGs with FDR $p$-value of $<0.05$ and $\log _{2}$ fold changes $>1$

in ovaries PBM (Suppl. Table 2). Differential expression of OBPs was also observed in An. gambiae mosquitoes between 24 and $48 \mathrm{~h}$ PBM [21], suggesting that mosquitoes are recovering their ability to respond to odors and/ or developing their ability to find good oviposition sites. Since we are studying the expression pattern in ovaries, these results suggest that ovaries may take part in the oviposition site selection or they may perform totally different functions.
Some of the induced OBPs are known to be involved in sensitive detection of oviposition attractants. For example, Culex quinquefasciatus OBP1 (orthologous to OBP56 in $A$. aegypti) not only binds to the mosquito oviposition pheromone, but is also involved in the reception of some oviposition attractants [45]. Our results showed that OBP56 and the ion channel ppk301 that controls freshwater egg-laying in $A$. aegypti were differentially expressed [46], (Suppl. Table 2). OBPs are also 


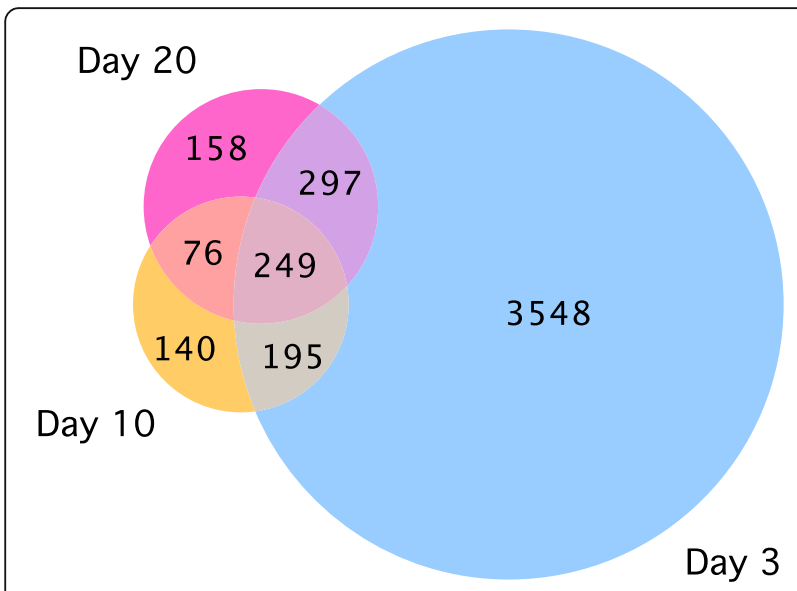

Fig. 3 Venn diagrams showing the number of differentially expressed genes (DEGs) between blood fed (BF) and sugar fed (SF) samples at three different time points. The numbers in the overlapping areas indicate genes that were common to both or to all three different time points. The number of DEGs was highest on day 3 PBM and 249 genes were differentially expressed at all three time points

expressed in the male reproductive tissues and transferred to the spermathecas of females [47]; OBPs thus may be involved in delivering pheromonal messages. It is also possible that OBPs are induced in response to the stress associated with oviposition or they may have a role in oocyte development. Additional studies are necessary to elucidate the roles of OPBs in ovaries PBM.

Several members of cytochrome P450 (CYP) family detoxification genes had altered expression patterns in the BF samples. Among the most and least DEGs, CYP325N2 had nearly 14-fold overexpression and CYP325N1 had 2-fold under-expression (Table 2). Four glutathione transferase genes exhibited 2-4 fold overexpression in the BF samples. On day 3 PBM, a large number of defense-related genes had a differential expression pattern (Table 3). TOLL was enriched, 20 CLIP genes were up and 2 were down, 12 LRIM were up; Defensin genes, GNBP genes, and Cecropin genes were over expressed following blood meal ingestion. HOP, DOME, and $I M D$ expressions were not significantly different. On day 10, TOLL5, 3 CLIP and 3 LRIMs were upregulated. On day 20, few more defense-specific genes compare to day 10 were differentially expressed (Table 3 ).

The overexpression of several detoxification enzymes suggests that blood-meal ingestion not only induces gene expression for egg development, but also prepares ovaries to deal with the ill effects of any bloodassociated toxins or its metabolites or to counter contamination by toxic environmental compounds during oviposition. In addition, expression of various defenseassociated genes was induced following the blood meal. These results were also supported by gene ontology analysis where oxidoreductase genes were found to be highly enriched (Fig. 4 and Supplementary Table 3). Akbari et al. [25] made a similar observation in PBM ovaries. Since blood is the primary source of infectious agents, such as viruses and pathogenic bacteria, ovaries become prepared to thwart pathogens from infecting germ-line cells. In a previous study, it was observed that several immunity-related transcripts accumulated at a lower level in blood-fed mosquitoes 5 h PBM [22]. Gene expression in ovaries occurs in waves following a blood meal [21, 25]. Genes that are up- or down-regulated early in the gonotrophic cycle are not the same that occur later during egg development. It is possible that changes in expression at the whole-body level may conceal the tissue-specific changes [22], or the defense related genes are induced later in the gonotrophic cycle. It is likely that slightly overexpressed genes on day 3 could be leftover RNAs from high levels of overexpression early in the gonotrophic cycle. The expression of defense-associated genes may also result from oviposition stress or to protect the reproductive tissues from becoming infected during oviposition. Expression of immunity genes PBM is strain dependent [48, 49], which may relate to the variability of vector competence for arboviruses observed in different geographic populations of $A$. aegypti. Arbovirus infections of ovaries from infectious blood meals occur late, usually long past the gonotrophic cycle [12]. The expression of immunity genes in ovaries PBM may be one of the reasons that ovary infections occur late. It would be interesting to see the ovary's response to an infectious blood meal.

During the PBM period, there are extreme physiological changes that require rapid coordination between tissues and between cells within the tissue. Intercellular channels, known as gap junctions, aid in the coordination of cells within tissues by the direct transfer of small molecules and ions between cells. In A. aegypti, six innexin genes (inx $1-4,7$, and 8 ) encode proteins that work as gap junctions. Similar to previous observations [50], we observed that several inx genes are differentially expressed (Suppl. Table 2). Among them, inx2 was most differentially expressed with 4-fold overexpression. Three cysteine-rich venom proteins were over-enriched in day 3 samples in BF ovaries. However, their expression levels were not enriched at later time points. These venom proteins are found in animal venoms acting on ion channels [51]. One of them (AAEL000379) is also differentially expressed in $A$. aegypti following Zika virus infection [41].

On days 10 and 20 PBM, most of the genes that had an altered expression pattern on day 3 in BF samples exhibited no significantly different expression patterns compared to SF samples. For example, among the OBPs, only OBP15 had 2-fold 
Table 1 Differentially expressed odorant binding proteins (OBPs)

\begin{tabular}{|c|c|c|c|c|}
\hline \multirow{2}{*}{ Gene } & \multirow{2}{*}{ Transcript ID } & \multicolumn{3}{|c|}{${ }^{\mathrm{a}}$ Fold change in expression } \\
\hline & & Day 3 & Day 10 & Day 20 \\
\hline OBP46 & AAEL010872-RA & $244(1.25 \mathrm{E}-08)$ & NSD & NSD \\
\hline OBP40 & AAEL009597-RA & $122(0.0136)$ & NSD & NSD \\
\hline OBP41 & AAEL009599-RA & $102(0.0168)$ & NSD & NSD \\
\hline OBP10 & AAEL007603-RA & $19(1.28 \mathrm{E}-11)$ & NSD & NSD \\
\hline OBP23 & AAEL006109-RA & $11(0.0013)$ & NSD & NSD \\
\hline OBP1 & AAEL006454-RA & $5(0.0367)$ & NSD & NSD \\
\hline OBP35 & AAEL002606-RA & $5(0.0029)$ & NSD & NSD \\
\hline OBP33 & AAEL006385-RB & $4(0.0273)$ & NSD & NSD \\
\hline OBP55 & AAEL012377-RB & $3(0.0165)$ & NSD & NSD \\
\hline OBP6 & AAEL000821-RC & $3(0.0006)$ & NSD & NSD \\
\hline OBP15 & AAEL002598-RA & $3(5.55 \mathrm{E}-06)$ & $2(0.0022)$ & NSD \\
\hline OBP22 & AAEL005772-RA & $3(0.0084)$ & NSD & NSD \\
\hline OBP56 & AAEL013018-RA & $-13(0.0114)$ & NSD & NSD \\
\hline OBP52 & AAEL011491-RB & NSD & NSD & $2(0.0478)$ \\
\hline
\end{tabular}

overexpression in the day 10 sample (Table 1). Among the five differentially expressed CYP genes on day 10, four had 2-fold and one had 5-fold overexpression (Table 2). No gap junction genes had significantly altered expression patterns on days 10 and 20. Defense-related genes showed a similar trend on days 10 and 20. However, there were few more defense-specific genes differentially expressed at day 20 than at day 10 . This late expression pattern of defense-associated genes could represent a response to environmental contamination or simply be due to aging.

\section{Gene ontology (GO)}

All DEGs were subject to gene ontology analysis using Blast2GO plug-in tool of the CLC workbench. Using this analysis tool, 93, 46, and 30 gene ontology (GO) terms were identified on days 3, 10 and 20, respectively (Suppl. Table 3). These GO terms were categorized into Biological process, Molecular function, and Cellular components. The enriched GO terms included peptidase activity, oxidoreductase activity, extracellular space, and hydrolase activity acting on glycosyl bonds, among others on day 3 (Fig. 4; Suppl. Table 3). There were 83 depleted GO functional terms, including ion binding, cell differentiation, signal transduction, cell death, and plasma membrane on day 3 (Suppl. Table 3). Highly significant top 10 downregulated categories are shown in Fig. 4. On days 10 and 20, enriched GO term categories were identical: peptidase activity and extracellular region. The depleted GO term categories were also similar at these two time points (Suppl. Table 3). These results suggest that mosquitoes are ready for another blood meal. 
Table 2 Differential expression of cytochrome P450 genes

\begin{tabular}{|c|c|c|c|c|}
\hline \multirow{2}{*}{ Gene } & \multirow{2}{*}{ Transcript ID } & \multicolumn{3}{|c|}{${ }^{2}$ Fold changes in expression on } \\
\hline & & Day 3 & Day 10 & Day 20 \\
\hline CYP325N2 & AAEL012762-RB & $13.88(1.56 \mathrm{E}-08)$ & & \\
\hline CYP325G2 & AAEL012766-RA & $12.3(0.0069)$ & & \\
\hline CYP6M9 & AAEL017297-RB & $11.13(0)$ & $2.41(0.0015)$ & $2.25(0.0043)$ \\
\hline СYР9J29 & AAEL014610-RA & $9.63(0.0085)$ & & \\
\hline CYP6M5 & AAEL009117-RA & $9.42(0)$ & & \\
\hline CYP9M6 & AAEL001312-RA & $7.44(0)$ & & $2.32(2.0 \mathrm{E}-08)$ \\
\hline CYP6Z9 & AAEL009129-RB & $6.87(0)$ & & $2.14(5.7 \mathrm{E}-06)$ \\
\hline CYP6AK1 & AAEL004941-RB & $6.79(2.21 \mathrm{E}-08)$ & & \\
\hline CYP9J22 & AAEL014619-RA & $6.44(1.60 \mathrm{E}-10)$ & & \\
\hline CYP6M10 & AAEL009125-RB & $6.35(0)$ & & \\
\hline CYP9J9 & AAEL014605-RA & $6.29(0)$ & & \\
\hline CYP9M9 & AAEL001807-RB & $6.15(0.0001)$ & & \\
\hline CYP9J28 & AAEL014617-RA & $5.7(1.19 \mathrm{E}-08)$ & & \\
\hline CYP9J2 & AAEL006805-RA & $5.2(0)$ & & $2.35(1.6 \mathrm{E}-07)$ \\
\hline CYP12F7 & AAEL002031-RB & $4.17(2.53 \mathrm{E}-12)$ & & \\
\hline CYP6N12 & AAEL009124-RA & $4.11(0.0128)$ & $5.6(0.0443)$ & \\
\hline СYР9J26 & AAEL014609-RA & $3.9(0.0051)$ & & \\
\hline CYP9J16 & AAEL006815-RA & $3.77(1.36 \mathrm{E}-12)$ & & \\
\hline CYP6Z6 & AAEL009123-RA & $3.73(8.44 \mathrm{E}-06)$ & & \\
\hline CYP315A1 & AAEL011850-RA & $3.59(1.53 \mathrm{E}-13)$ & $2.9(1.23 \mathrm{E}-06)$ & $2.33(0.0002)$ \\
\hline CYP6AH1 & AAEL007473-RA & $3.37(1.50 \mathrm{E}-06)$ & & \\
\hline CYP6N6 & AAEL009126-RA & $3.32(2.30 \mathrm{E}-06)$ & & \\
\hline CYPGN9 & AAEL009121-RA & $3.14(7.90 \mathrm{E}-08)$ & & \\
\hline CYP6M6 & AAEL009128-RA & $3.11(2.18 \mathrm{E}-10)$ & $2.17(0.0013)$ & \\
\hline CYP18A1 & AAEL004870-RA & $3.07(7.42 \mathrm{E}-10)$ & & \\
\hline CYP6N16 & AAEL010151-RB & $2.96(2.23 \mathrm{E}-13)$ & & \\
\hline CYP6P12 & AAEL014891-RB & $2.92(0)$ & & \\
\hline CYP6AG4 & AAEL007010-RA & $2.89(0.0091)$ & & \\
\hline CYP4C50 & AAEL008017-RA & $2.73(0.0087)$ & & \\
\hline CYP6S3 & AAEL009120-RA & $2.72(0.0349)$ & & \\
\hline CYP305A5 & AAEL002043-RA & $2.7(2.17 \mathrm{E}-10)$ & & \\
\hline СYР9J17 & AAEL006784-RA & $2.65(0.0007)$ & & \\
\hline CYP4D39 & AAEL007808-RA & $2.57(0.0004)$ & & \\
\hline CYP325E3 & AAEL000338-RB & $2.53(0.0055)$ & & \\
\hline CYP6N13 & AAEL009137-RA & $2.48(0.0023)$ & $2.26(0.0446)$ & \\
\hline CYP4D23 & AAEL007816-RA & $2.47(8.55 \mathrm{E}-07)$ & & \\
\hline CYP6Z8 & AAEL009131-RA & $2.46(0.0261)$ & & \\
\hline CYP6Z7 & AAEL009130-RA & $2.11(0.0313)$ & & \\
\hline CYP9AE1 & AAEL003748-RA & $2.1(0.0003)$ & & \\
\hline CYP325N1 & AAEL012770-RA & $-2.28(0.0002)$ & & \\
\hline CYP325S2_1 & AAEL019910-RA & $-2.76(0.0233)$ & & \\
\hline CYP307A1 & AAEL009762-RB & $-3.63(4.07 \mathrm{E}-13)$ & & \\
\hline CYP325G3 & AAEL012772-RA & $-3.86(0.0033)$ & & \\
\hline CYP325X1 & AAEL005695-RA & & & $2.76(0.0482)$ \\
\hline CYP325M5 & AAEL011761-RA & & & $2.75(0.0168)$ \\
\hline
\end{tabular}


Table 3 Differentially expressed defense-specific genes post blood meal

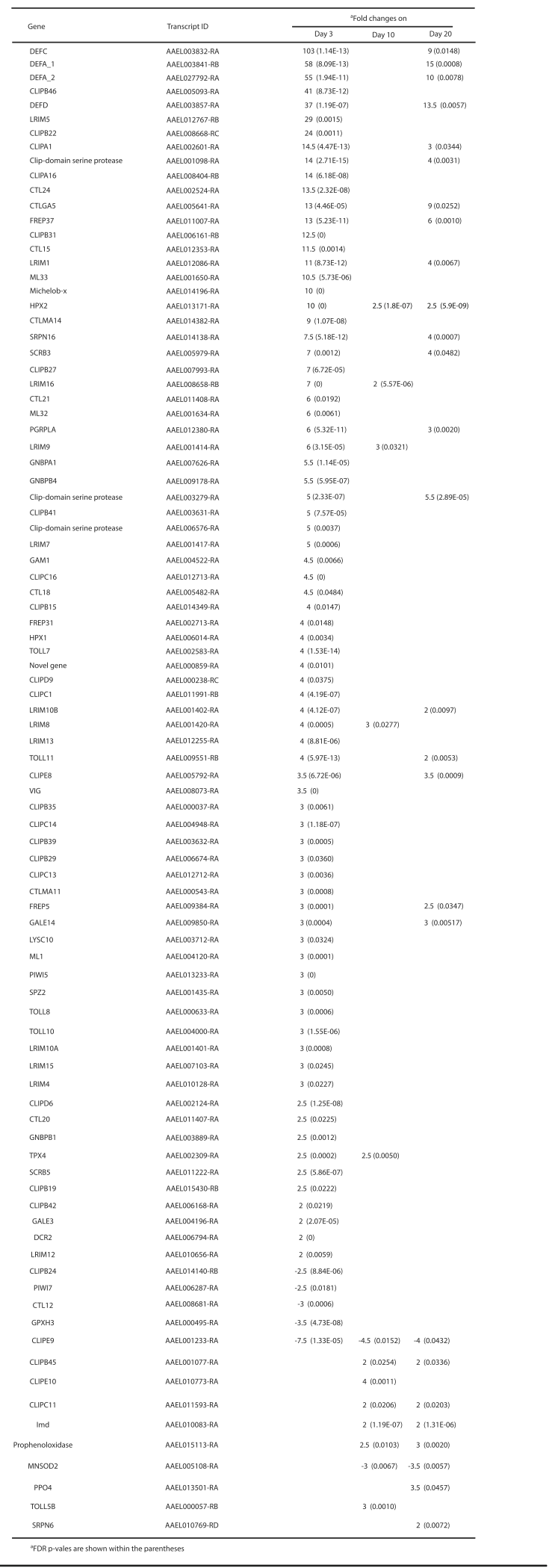

Differentially expressed long noncoding RNAs

In addition to the large number of differentially expressed protein-coding genes, we identified a large number of long non-coding RNAs (LncRNAs) in all three time points. LncRNAs are mRNA-like transcripts longer than 200 nucleotides but do not encode proteins. The functions of the LncRNAs, however, remain largely unknown, with a few exceptions that include LncRNAs with defined roles in embryogenesis, development, dosage compensation and sleep behavior, and they are also implicated in virus-host interactions [52-59]. The highest number of LncRNAs was observed on day 3 PBM (Suppl. Table 4). Among 2743 DEGs, 495 (18\%) were LncRNAs. At 10 and 20 days PBM, the corresponding numbers were $11 \%(41 / 363)$ and $10 \%$ (44/436), respectively. Fold changes in the expression level ranged from 660-fold increase to an 83-fold decrease on day 3 PBM. Thirteen LncRNA were common in all three time points (Fig. 5). LncRNAs were also found to be differentially expressed in $A$. aegypti mosquitoes following ingestion of a Zika virus containing infectious blood meal [41]. It would be interesting to determine if these LncRNAs have any roles in egg development, embryogenesis, or in defense against pathogens.

\section{RNA-Seq validation}

As mentioned above, gap-junction proteins were differentially expressed in our system as in previous studies [50]. Expression levels of genes (the ZIPs and ZnTs) encoding proteins that transport iron across membranes are increased in ovaries following a blood meal [60]. Our studies also showed that the expression of AAEL014762 (a ZIP family member) was increased nearly two-fold (FDR $p$-value $2.71 \times 10^{-15}$ ).

In addition, levels of 10 random transcripts showing differential expression PBM were tested by real-time quantitative polymerase chain reaction (RT-qPCR) (Table 4). Primers used are described in Supplementary Table 5. Transcripts were randomly chosen to include genes that showed either an enhanced or a decreased expression level. Among the genes tested, although the level of expression change does not match with that of the RNA-Seq analysis, the overall trend (over or underexpression) remained the same in both RT-qPCR and RNA-Seq analyses. Among the 10 genes tested, we included OBP46, an odorant-binding protein, to confirm that OBPs were differentially expressed following a blood meal in ovaries. OBP46 was overexpressed in both RNA-Seq and qPCR analysis. Our RT-qPCR results, thus, confirmed the RNA-Seq results (Table 4).

Most of the DEGs on day 3 returned to the nonsignificant level at later time points. These results were expected since most of the mosquitoes have laid their eggs by day 10 and are ready for a second blood meal. 


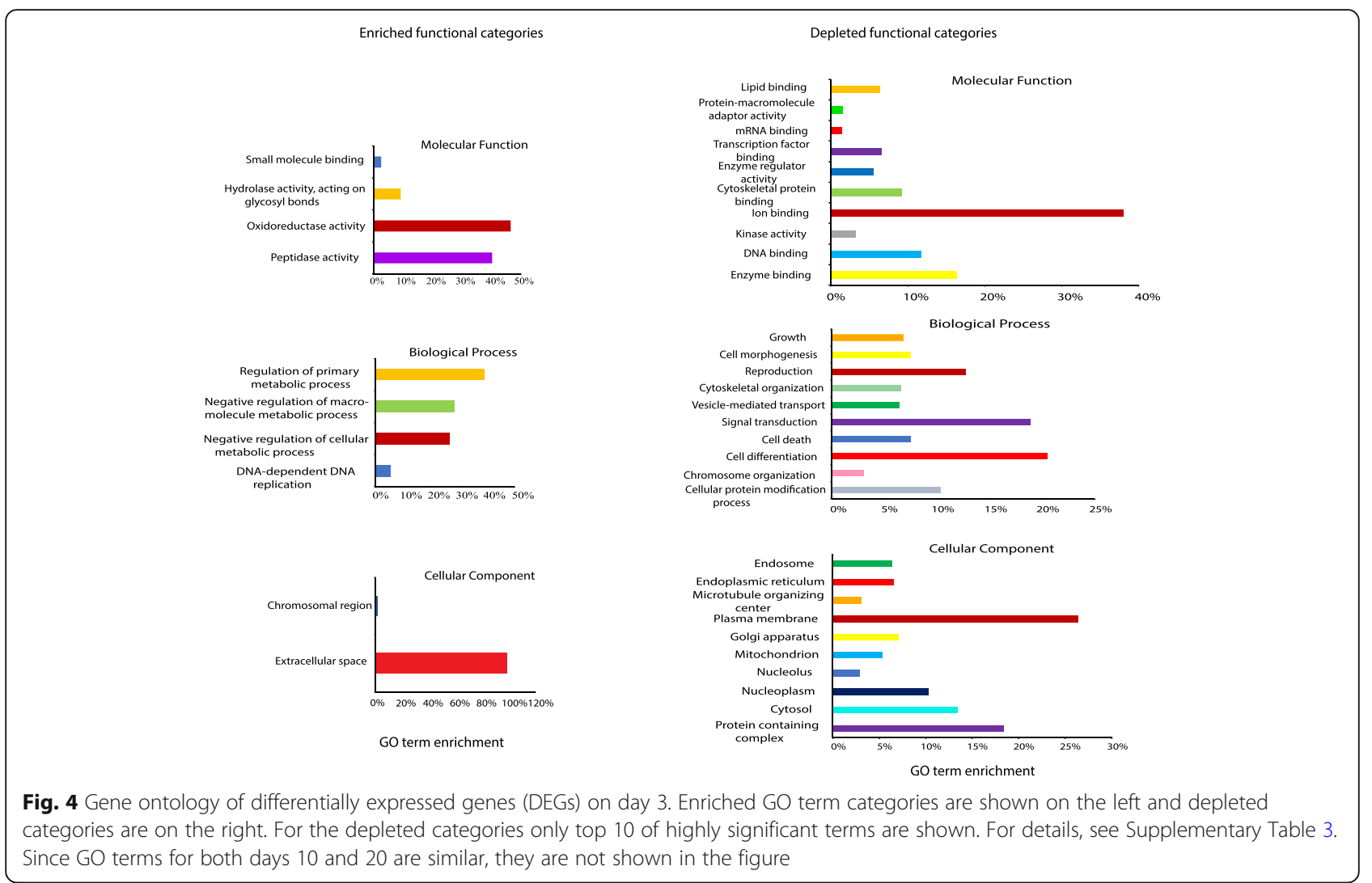

Accordingly, gene ontology analysis confirmed that both days 10 and 20 had similar enriched and depleted gene ontology term categories. For example, both peptidase activity and extracellular region categories were enriched on both days 10 and 20 . The result of our gene-ontology analysis is also similar to that of the gene expression studies by EST analysis in autogenous Georgecraigius

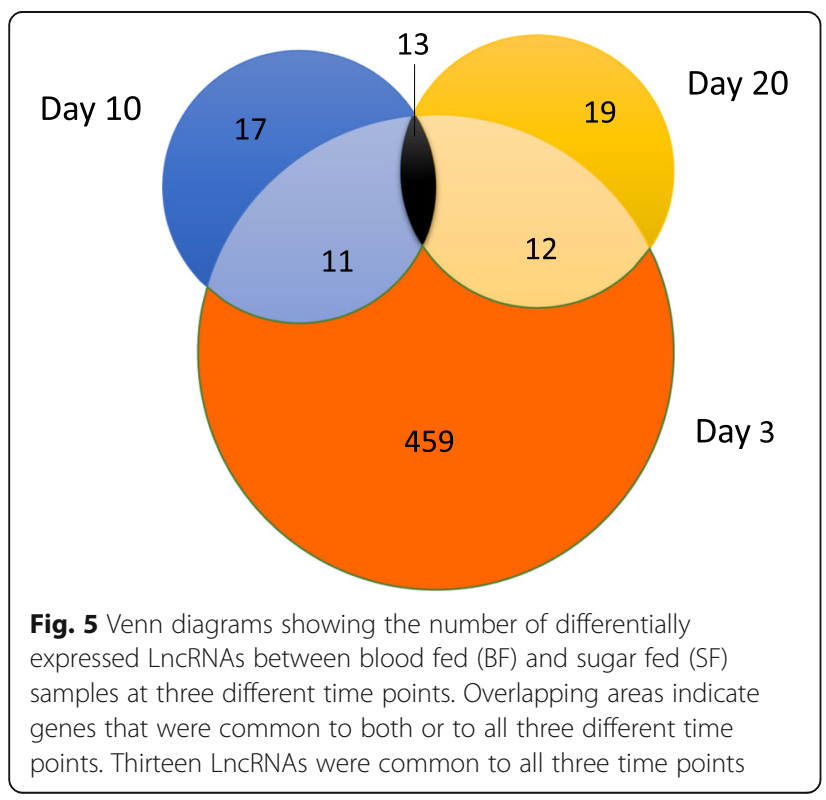

atropalpus mosquito ovaries during the autogenous phase of egg production [23]. Similarities include identification of transcripts associated with intermediary metabolism and extracellular space (Suppl. Table 3).

Although most DEGs returned to the non-significant level at later time points, the gene expression pattern in blood-fed mosquito ovaries differed slightly from SF ovaries at the same time point. More than 300 genes exhibited differential expression patterns on days 10 and 20 PBM. The data presented here represent ovary transcriptomes in mosquitoes that do not take a second blood meal during a gonotrophic cycle. In nature, A. aegypti generally takes multiple blood meals during a single gonotrophic cycle. Each meal will trigger gene expression for egg maturation. Consequently, the pattern of gene expression observed in our studies may not represent what happens in nature. Further studies with additional blood feeding in the same gonotrophic cycle are necessary to address this issue.

\section{Conclusions}

A large number of genes were differentially expressed following ingestion of a blood meal in mosquito ovaries when compared to SF ovaries. Differential expression is highest on day 3 PBM and most of the DEGs return to the non-significant level by day 10 , although expression was not identical to the SF samples. Major categories of 
Table 4 Quantitative PCR validation of the RNA-Seq ressults

\begin{tabular}{|c|c|c|c|c|c|c|c|}
\hline \multirow{3}{*}{ Gene } & \multirow{3}{*}{ Transcript } & \multicolumn{2}{|l|}{ Day 3} & \multicolumn{2}{|l|}{ Day 10} & \multicolumn{2}{|l|}{ Day 20} \\
\hline & & \multicolumn{2}{|c|}{ Fold change $\mathrm{e}^{\mathrm{a}, \mathrm{b}}$} & \multicolumn{2}{|c|}{ Fold change } & \multicolumn{2}{|c|}{ Fold change } \\
\hline & & RNAseq & $2^{-\Delta \Delta c_{T}}$ & RNAseq & $2^{-\Delta \Delta C_{T}}$ & RNAseq & $2^{-\Delta \Delta C_{T}}$ \\
\hline 4-hydroxytryptamine kinase & AAEL019856-RA & $272(0) \uparrow$ & $308 \uparrow$ & $7(4.74 \mathrm{E}-05) \uparrow$ & $8.28 \uparrow$ & $4.4(0.0021) \uparrow$ & $5.98 \uparrow$ \\
\hline Hypothetical protein & AAEL005192-RA & $658(0) \uparrow$ & $3 \times 10^{5} \uparrow$ & $18(1.82 \mathrm{E}-05) \uparrow$ & $445 \uparrow$ & $25(6.77 \mathrm{E}-07) \uparrow$ & $69.60 \uparrow$ \\
\hline Hypothetical protein & AAEL008417-RA & $22(9.58 \mathrm{E}-06) \downarrow$ & $1.21 \downarrow$ & NS & & NS & \\
\hline Hypothetical protein & AAEL023200-RA & $273(0.0048) \quad \uparrow$ & $2.01 \uparrow$ & NS & & NS & \\
\hline Alcohol dehydrogenase & AAEL017418-RA & $11(3.39 \mathrm{E}-05) \downarrow$ & $4.14 \downarrow$ & $11(0.0006) \downarrow$ & $4.19 \downarrow$ & NS & \\
\hline Hypothetical protein & AAEL004130-RA & NS & & $5(0.009) \downarrow$ & $3.63 \downarrow$ & $11(9.2 \mathrm{E}-06) \downarrow$ & $7.51 \downarrow$ \\
\hline Hypothetical protein & AAEL006138-RA & $9(8.84 \mathrm{E}-11) \uparrow$ & $4.43 \uparrow$ & $16(1.65 \mathrm{E}-11) \uparrow$ & $2.71 \uparrow$ & $19(1.05 \mathrm{E}-11) \uparrow$ & $3.50 \uparrow$ \\
\hline Hypothetical protein & AAEL020455-RA & NS & & $10(7.92 \mathrm{E}-06) \downarrow$ & $2.34 \downarrow$ & NS & \\
\hline Hypothetical protein & AAEL013218-RA & $400(0) \uparrow$ & $280 \uparrow$ & NS & & NS & \\
\hline OBP46 & AAEL010872-RA & $244(1.25 \mathrm{E}-08) \uparrow$ & $33.60 \uparrow$ & NS & & NS & \\
\hline
\end{tabular}

${ }^{\mathrm{a} F D R} \mathrm{p}$-values are shown within parentheses, ${ }^{\mathrm{b}}$ Arrowheads indicate either over or under-expression. Ns, Non-significant.

DEGs on day 3 included OBPs, cytochrome P-450 mediated detoxification genes, defense-specific genes among others. Gene ontology terms including, peptidase activity, oxidoreductase activity, negative regulation of cellular metabolic and macromolecule metabolic processes were significantly enriched on day 3 PBM. Since blood is the primary source of infection by viral or other infectious agents, ovaries must become prepared to deal with blood-borne or environmental pathogens to protect their germ-line cells. Studies are underway to reveal ovary's response to infectious blood meals.

\section{Methods}

\section{Mosquito feeding and rearing}

All mosquito rearing was done at $28^{\circ} \mathrm{C}$. 4-7 days old $A$. aegypti (from Poza Rica, Mexico, kindly provided by Greg Ebel, CSU) were fed with warmed sheep blood (Colorado Serum Company). No additional blood meals were given for the entire study period. After feeding for an hour at room temperature, engorged females were separated in cardboard containers. A $65-\mathrm{mm}$ petri dish containing water-soaked cotton covered with a wet coffee filter paper was placed inside the jar for laying eggs. All mosquitoes, both control and blood fed, were maintained with $10 \%$ sucrose and reared identically.

\section{Library preparation}

Ovary samples were collected on days 3, 10, and 20 PBM. Ovary dissection was carried out in sterile $1 \mathrm{X}$ phosphate buffered saline and stored immediately at $-80{ }^{\circ} \mathrm{C}$ until RNA isolation. Ovaries were collected from about 25 mosquitoes for each time point. Eggs, if present, were removed from the ovaries before collection. Total RNA was isolated using the mirVana miRNA isolation kit (Invitrogen) then treated with Turbo DNase followed by inactivation of the DNase using the supplied DNase inactivation reagent following the manufacturer's suggestions. rRNA was depleted using the Illumina Ribo-Zero gold kit. Prior to library preparation, mosquito total RNA was quality checked using an Agilent Fragment Analyzer and Qubit Fluorescence (Invitrogen). RNA libraries were prepared following the Illumina Stranded total RNA library prep kit with Ribo-Zero Gold protocol. Following library concentration and quality control, final libraries were pooled to $10 \mathrm{nM}$ and the concentration of the pool was determined using the Kapa Biosystems Universal qPCR kit. After dilution and denaturing, the pooled library was loaded onto a NovaSeq6000 S1 flow cell (PE200) for sequencing. Library preparation and sequencing were done with three biological replicates at the University of Buffalo Genomics and Bioinformatics Core. A total of 19 samples were sequenced. Eighteen samples from three biological replicates (Replicates 1, 2, and 4). There was room for an additional sample; we included a day 3 blood-fed sample from another replicate (Replicate 3 ). The raw sequences were subjected to quality check by FastQC.

\section{RNA-Seq data analysis}

Qiagen CLC genomics workbench v.20 was used for bioinformatics analysis in this study. Compressed FASTQ files were extracted, trimmed, and filtered using the 
default parameters of the modified Mott trimming algorithm as implemented in CLC. Differential gene expression analyses were performed using the proprietary RNAseq analyses module as implemented in Qiagen CLC genomics workbench using the default parameters. The CLC RNAseq workflow uses multi-factorial statistics based on a negative binomial Generalized Linear Model (GLM) to determine statistically supported differentially expressed genes between treatments. More specifically, reads were mapped to the VectorBase reference genome (Aedes aegypti L 5.0) with mismatch, insertion, and deletion costs parameters set at 2,3 , and 3, respectively, with a length fraction of 0.8 in mapping parameter, where $80 \%$ of the nucleotides in a read must be aligned to the reference genome. Principal component analysis (PCA) was used for quality control, tissue contamination, problems with experimental design, and to visualize variation between expression analysis samples. Venn diagram and volcano plot were generated on the CLC workbench.

\section{Gene ontology}

Functional annotation and gene ontology analysis were carried out using the Blast2go Plug-in tool of the CLC workbench. We used blast (SwissProt) and InterProscan to reveal the gene ontology term for the A. aegypti transcripts obtained from VectorBase (Aedes-aegypti- LVP AGWG_TRANSCRIPTS_AaegL5.2.fa.gz). Fisher's exact test was used to categorize the DEGs into Biological process, Molecular function, and Cellular components.

\section{RT-qPCR analysis of mRNA}

To validate the RNA-Seq results, we used RT-qPCR to measure the relative abundance of 10 transcripts in ovary total RNA preparations using RNA samples from two biological replicates used in the RNA-Seq analysis and two new biological replicates. So, RNA samples from four replicates were used for RT-qPCR validation. Total RNA was prepared and treated with Turbo DNase as described above. Total RNA was reverse transcribed (200 ng in a $20 \mu \mathrm{l}$ total volume) using superscript IV first strand synthesis system (Thermofisher Scientific). A mixture of Oligo $\mathrm{d}(\mathrm{T})_{20}$ and random hexamers was used as primers. Real time Quantitative PCR was performed using $1 \mathrm{X}$ powerup SYBR green master mix and $4 \mathrm{pM}$ of each primer and $0.2 \mu \mathrm{l}$ of the cDNA mixture in a $10-\mu \mathrm{l}$ total volume. Relative abundance was determined using the Livak $\left(\Delta \Delta C_{\mathrm{T}}\right)$ method. All polymerase reactions were performed in quadruplicate. The ribosomal S7 proteincoding gene was used as an endogenous reference. Primer sequences are shown in supplementary Table 5.

\section{Abbreviations}

BF: Blood fed; CYP: Cytochrome P450; DEG: Differentially expressed gene;

EST: Expressed sequence tag; FDR: False discovery rate; LnCRNA: Long non- coding RNA; OBP: Odorant binding protein; PBM: Post blood meal; PCA: Principal component analysis; RT-qPCR: Reverse-transcriptase quantitative polymerase chain reaction; RNA-Seq: RNA sequencing; SF: Sugar fed

\section{Supplementary Information}

The online version contains supplementary material available at https://doi. org/10.1186/s12864-021-07551-z

Additional file 1: Table S1. RNA read summary of BF and SF libraries.

Additional file 2: Table S2. Significant DEGs between BF and SF samples at three different time points.

Additional file 3: Table S3. Functional categories of DEGs.

Additional file 4: Table S4. Differential expression of LncRNAs at three time points.

Additional file 5: Table S5. Primers used for qPCR assay.

\section{Acknowledgements}

We thank University of Buffalo Genomics and Bioinformatics Core for library preparation and sequencing work, and Wadsworth Center's Bioinformatics and Statistics core for preliminary bioinformatics analysis. The Wadsworth Center's Media and Tissue culture facility provided vero, mosquito cells, and the necessary media. The authors would like to thank the insectary staff for maintaining mosquito colonies and providing mosquito eggs, and Anne Payne for help with the ovary dissection. DKN would like to thank Eric Seiser of Qiagen Bioinformatics for help with the CLC workbench bioinformatics analysis. Finally, we would like to thank the anonymous Reviewers' for their suggestions that have improved the manuscript significantly.

\section{Authors' contributions}

DKN and LDK conceived the idea. DKN designed the experiments; DKN and CD conducted the experiments, DKN analyzed the data, DKN, PL, and EL-N carried out the bioinformatics analysis; DKN, LDK and PL wrote/edited the manuscript. All authors read and approved the final manuscript.

\section{Funding}

This work was supported by the NIH grant 1 R21 Al138344 (DKN and LDK). CD was partially supported by the Cooperative Agreement Number U01CK000509 funded by the Centers for Disease Control and Prevention (LDK). The funding bodies had no role in the design of the study, and collection, analysis and interpretation of the data and writing of the manuscript.

\section{Availability of data and materials}

All raw and processed data are deposited at NCBI Gene Expression Omnibus (GEO) with the accession number GEO GSE152209 (https://www.ncbi.nlm.nih. gov/gds/?term=GEO\%20GSE152209). Gene set annotation, expression data and identified long non-coding RNAs are included as supplementary files with the manuscript.

\section{Declarations}

Ethics approval and consent to participate

Not applicable.

\section{Consent for publication}

Not applicable.

\section{Competing interests}

The authors declare that they have no competing interests.

\section{Author details}

${ }^{1}$ Arbovirus Laboratory, Wadsworth Center, New York State Department of Health, Slingerlands, NY 12159, USA. ${ }^{2}$ Bioinformatics Core, Wadsworth Center, New York State Department of Health, Center for Medical Science, Albany, NY 12208, USA. ${ }^{3}$ Department of Biomedical Sciences, State University of New York, School of Public Health, Albany, NY 12208, USA. 
Received: 6 July 2020 Accepted: 24 March 2021

\section{Published online: 27 May 2021}

\section{References}

1. Gubler DJ. Vector-borne diseases. Rev Sci Tech. 2009:28:583-8.

2. World health Organization. Global vector control response 2017-2030. WHO Press 2017.

3. Clements AN. The biology of mosquitoes. London: Chapman and Hall; 1992.

4. Tesh RB. Transovarial transmission of arboviruses in their invertebrate vectors. In: Harris KF, editor. Current topics in vector research. New York: NY; 1984. p. $57-76$.

5. Leake CJ. Transovarial transmission of arboviruses by mosquitoes. Vectors in virus biology. New York and London: Academic Press; 1984. p. 63-91.

6. Rosen L. Sexual transmission of dengue viruses by Aedes albopictus. Am J Trop Med Hyg. 1987;37(2):398-402.

7. Lipsitch M, Siller S, Nowak MA. The evolution of virulence in pathogens with vertical and horizontal transmission. Evolution. 1996;50:1729-41.

8. Saiyasombat R, Bolling BG, Brault AC, Bartholomay LC, Blitvich BJ. Evidence of efficient transovarial transmission of Culex flavivirus by Culex pipiens (Diptera: Culicidae). J Med Entomol. 2011;48(5):1031-8.

9. Lequime S, Paul RE, Lambrechts L. Determinants of arbovirus vertical transmission in mosquitoes. PLoS Pathog. 2016;12(5):e1005548.

10. Thangamani S, Huang J, Hart CE, Guzman H, Tesh RB. Vertical transmission of Zika virus in Aedes aegypti mosquitoes. Am J Trop Med Hyg. 2016;95(5): 1169-73.

11. Ciota AT, Bialosuknia SM, Ehrbar DJ, Kramer LD. Vertical transmission of Zika virus by Aedes aegypti and Ae. albopictus mosquitoes. Emerg Infect Dis. 2017;23(5):880-2.

12. Sánchez-Vargas I, Harrington LC, Doty JB, Black WC, Olson KE. Demonstration of efficient vertical and venereal transmission of dengue virus type-2 in a genetically diverse laboratory strain of Aedes aegypti. PLoS Negl Trop Dis. 2018;12(8):e0006754.

13. Briegel $H$. Fecundity, metabolism, and body size in Anopheles (Diptera: Culicidae), vectors of malaria. J Med Entomol. 1990;27:839-50.

14. Dana AN, Hong YS, Kern MK, Hillenmeyer ME, Harker BW, Lobo NF, et al. Gene expression pattern associated with blood-feeding in the malaria mosquito Anopheles gambiae. BMC Genomics. 2005;6:5.

15. Baker FC, Hagedorn HH, Schooley DA, Wheelock G. Mosquito juvenile hormone identification and bioassay activity. J Insect Physiol. 1983;29:465-70.

16. Racioppi JV, Gemmill RM, Kogan PH, Calvo JM, Hagedorn HH. Expression and regulation of vitellogenin messenger RNA in the mosquito, Aedes aegypti. Insect Biochem. 1986;16:255-62.

17. Raikhel AS, Dhadialla TS. Accumulation of yolk proteins in insect oocytes. Annu Rev Entomol. 1992;37:217-51.

18. Raikhel AS, Kokoza VA, Zhu J, Martin D, Wang SF, Li C, et al. Molecular biology of mosquito vitellogenesis: from basic studies to genetic engineering of antipathogen immunity. Insect Biochem Mol Biol. 2002;32: $1275-86$.

19. Ozsolak F, Platt AR, Jones $D R$, Reifenberger JG, Sass LE, Mclnerney $P$, et al. Direct RNA sequencing. Nature. 2009;461:814-8.

20. Nagalakshmi U, Waern K, Snyder M. RNA-seq: a method for comprehensive transcriptome analysis. Curr Protoc Mol Biol. 2010; 1-13, Chapter 4: unit 4.11.

21. Marinotti O, Calvo E, Nuyen QK, Dissanayake S, Ribeiro JMC, James AA Genome-wide analysis of gene expression in adult Anopheles gambiae. Insect Mol Biol. 2006;15(1):1-12.

22. Bonizzoni M, Dunn WA, Campbell CL, Olson KE, Dimon M, Marinotti O, et al. RNA-seq analysis of blood-induced changes in gene expression in the mosquito vector species, Aedes aegypti. BMC Genomics. 2011;12:82.

23. Telang A, Rechel JA, Brandt JR, Donnell DM. Analysis of ovary-specific genes in relation to egg maturation and female nutritional condition in the mosquitoes Georgecraigius atropalpus and Aedes aegypti (Diptera: Culicidae). J Insect Physiol. 2013;59(3):283-94.

24. Rinker DC, Pitts RJ, Zhou XJ, Suh E, Rokas A, Zwiebel LJ. Blood meal-induced changes to antennal transcriptome profiles reveal shifts in odor sensitivities in Anopheles gambiae. Proc Natl Acad Sci U S A. 2013;110:8260-5.

25. Akbari OS, Antoshechkin I, Amrhein H, Williams B, Diloreto R, Sandler J, et al. The developmental transcriptome of the mosquito Aedes aegypti, an invasive species and major arbovirus vector. G3 (Bethesda). 2013;3(9): 1493-509.
26. Roy $S$, Saha TT, Johnson L, Zhao B, Ha J, White KP, et al. Regulation of gene expression patterns in mosquito reproduction. PLoS Genet. 2015;11(8): e1005450.

27. Matthews BJ, McBride CS, DeGennaro M, Despo O, Vosshall LB. The neurotranscriptome of the Aedes aegypti mosquito. BMC Genomics. 2016;17:32.

28. Taparia $T$, Ignell R, Hill SR. Blood meal induced regulation of the chemosensory gene repertoire in the southern house mosquito. BMC Genomics. 2017;18:393.

29. Gamez S, Antoshechkin I, Mendez-Sanchez SC, Akbari OS. The developmental transcriptome of Aedes albopictus, a major worldwide human disease vector. G3 (Bethesda). 2020;10(3):1051-62.

30. Xi Z, Ramirez JL, Dimopoulos G. The Aedes aegypti TOLL pathway controls dengue virus infection. PLoS Pathog. 2008;4:e1000098.

31. Sanchez-Vargas I, Scott JC, Poole-Smith BK, Franz AW, Barbosa-Solomieu V, Wilusz J, et al. Dengue virus type 2 infections of Aedes aegypti are modulated by the mosquito's RNA interference pathway. PLoS Pathog. 2009:5:e1000299.

32. Souza-Neto JA, Sim S, Dimopoulos G. An evolutionary conserved function of the JAK-STAT pathway in anti-dengue defense. Proc Natl Acad Sci U S A. 2009;106:17841-6.

33. Sim S, Dimopoulos G. Dengue virus inhibits immune responses in Aedes aegypti cells. PLoS One. 2010;5:e10678.

34. Tchankouo-Nquetcheu S, Khun H, Pincet L, Roux P, Bahut M, Huerre M, et al Differential protein modulation in midguts of Aedes aegypti infected with chikungunya and dengue 2 viruses. PLoS One. 2010;5:e13149.

35. Behura SK, Gomez-Machorro C, Harker BW, deBruyn B, Lovin DD, Hemme RR, et al. Severson DW Global cross-talk of genes of the mosquito Aedes aegypti in response to dengue virus infection. PLoS Negl Trop Dis. 2011;5: e1385.

36. Colpitts TM, Cox J, Vanlandingham DL, Feitosa FM, Cheng G, Kurscheid S, et al. Alterations in the Aedes aegypti transcriptome during infection with West Nile, dengue and yellow fever viruses. PLoS Pathog. 2011;7:e1002189.

37. Bonizzoni M, Dunn WA, Campbell CL, Olson KE, Marinotti O, James AA. Complex modulation of the Aedes aegypti transcriptome in response to dengue virus infection. PLoS One. 2012;7:e50512.

38. Chauhan C, Behura SK, Debruyn B, Lovin DD, Harker BW, Gomez-Machorro $C$, et al. Comparative expression profiles of midgut genes in dengue virus refractory and susceptible Aedes aegypti across critical period for virus infection. PLoS One. 2012;7:e47350

39. Sim S, Ramirez JL, Dimopoulos G. Dengue virus infection of the Aedes aegypti salivary gland and chemosensory apparatus induces genes that modulate infection and blood-feeding behavior. PLoS Pathog. 2012;8(3): e1002631.

40. Dong S, Behura SK, Franz AWE. The midgut transcriptome of Aedes aegypti fed with saline or protein meals containing chikungunya virus reveals genes potentially involved in viral midgut escape. BMC Genomics. 2017;18:382.

41. Etebari K, Hegde S, Saldana MA, Widen SG, Wood TG, Asgari S, et al. Global transcriptome analysis of Aedes aegypti mosquitoes in response to Zika virus infection. mSphere. 2017;2(6):200456-17.

42. Pelosi P, lovinella I, Felicioli A, Dani FR. Soluble proteins of chemical communication: an overview across arthropods. Front Physiol. 2014;5:320.

43. Leal WS. Odorant reception in insects: roles of receptors binding proteins, and degrading enzymes. Annu Rev Entomol. 2013;58:373-91.

44. Bonizzoni M, Dunn WA, Campbell CL, Olson KE, Marinotti O, James AA. Strain variation in the transcriptome of the dengue fever vector, Aedes aegypti. G3 (Bethesda). 2012;2:103-14.

45. Pelletier J, Guidolin A, Syed Z, Cornel AJ, Leal WS. Knockdown of a mosquito odorant-binding protein involved in the sensitive detection of oviposition attractants. J Chem Ecol. 2010;36:245-8.

46. Matthews BJ, Younger MA, Vosshall LB. The ion channel ppk301 control freshwater egg-laying in the mosquito Aedes aegypti. eLife. 2019;8:e43963.

47. Li S, Picimbon J-F, Ji S, Kan Y, Chuanling Q, Zhou J-J, et al. Multiple functions of an odorant-binding protein in the mosquito Aedes aegypti. Biochem Biophys Res Comm. 2008:372:464-8.

48. Bennet KE, Olson KE, Munoz ML, Fernandez-Salas I, Farfan-Ale JA, Higgs S, et al. Variation in vector competence for dengue 2 virus among 24 collections of Ae. aegypti from Mexico and the United States. Am J Trop Med Hyg. 2002;67:85-92.

49. Black WC, Bennet KE, Gorrochotegui-Escalante N, Barillasa-Mury CV, Fernandez-Salas I, Munoz ML, et al. Flavivirus susceptibility in Aedes aegypti. Arch Med Res. 2002;33:379-88. 
50. Calkins TL, Piermarini PM. A blood meal enhances innexin mRNA expression in the midgut, malpighian tubules, and ovaries of the yellow fever mosquito Aedes aegypti. Insects. 2007;8(4):122.

51. Gibbs GM, O'Bryan MK. Cystein rich secretory proteins in reproduction and venom. Soc Reprod Fertil Suppl. 2007;65:261-7.

52. Heard $E$, Disteche CM. Dosage compensation in mammals: fine-tuning the expression of the X chromosome. Genes Dev. 2006;20:1848-67.

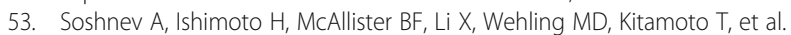
A conserved long noncoding RNA affects sleep behavior in Drosophila. Genetics. 2011;189:455-68.

54. Pauli A, Valen E, Lin MF, Garber M, Vastenhouw NL, Levin JZ, et al. Systematicidentification of long noncoding RNAs expressed during zebrafish embryogenesis. Genome Res. 2012;22:577-91.

55. Li M, Wen S, Guo X, Bai B, Gong Z, Liu X, et al. The novel long non-coding RNA CRG regulates Drosophila locomotor behavior. Nucleic Acids Res. 2012; 40:11714-27.

56. Lv J, Liu H, Huang Z, Su J, He H, Xiu Y, et al. Long non-coding RNA identification over mouse brain development by integrative modeling of chromatin and genomic features. Nucleic Acids Res. 2013;41:10044-61.

57. Mercer TR, Mattick JS. Structure and function of long noncoding RNAs in epigenetic regulation. Nat Struct Mol Biol. 2013;20:300-7.

58. Etebari K, Asad S, Zhang G, Asgari S. Identification of Aedes aegypti long intergenic non-coding RNAs and their association with Wolbachia and dengue virus infection. PLoS Negl Trop Dis. 2016;10:e0005069.

59. Winterling C, Koch M, Koeppel M, Garcia-Alcalde F, Karlas A, Meyer TF. Evidence for a crucial role of host non-coding RNA in influenza a virus replication. RNA Biol. 2014;11:66-75.

60. Tsujimoto $\mathrm{H}$, Anderson MAE, Myles KM, Adelman ZN. Identification of candidate iron transporters from the $\mathrm{ZIP} / \mathrm{ZnT}$ gene families in the mosquito Aedes aegypti. Front Physiol. 2018;9:380.

\section{Publisher's Note}

Springer Nature remains neutral with regard to jurisdictional claims in published maps and institutional affiliations.

Ready to submit your research? Choose BMC and benefit from:

- fast, convenient online submission

- thorough peer review by experienced researchers in your field

- rapid publication on acceptance

- support for research data, including large and complex data types

- gold Open Access which fosters wider collaboration and increased citations

- maximum visibility for your research: over $100 \mathrm{M}$ website views per year

At $\mathrm{BMC}$, research is always in progress.

Learn more biomedcentral.com/submissions 